[Article]

\title{
单分散 $\mathrm{SnO}_{2}$ 中空微纳米球的制备和性质
}

\author{
蔡宏敏 ${ }^{1}$ 任素贞 ${ }^{1, *}$ 王 萌 ${ }^{1}$ 贾翠英 ${ }^{2}$ \\ ('大连理工大学化学学院, 辽宁大连 116024; ${ }^{2}$ 大连理工大学化学实验中心, 辽宁大连 116024)
}

\begin{abstract}
摘要: 模板法是制备无机中空微纳米球的重要方法之一. 本文以苯乙烯为单体, 通过乳液聚合得到粒径约为 $620 \mathrm{~nm}$ 的单分散聚苯乙烯(PS)微球. 以磺化后的聚苯乙烯(PSS)微球为模板, 利用阴阳离子静电吸附作用, 将 $\mathrm{PSS}$ 与前驱体 $\mathrm{SnSO}_{4}$ 中的 $\mathrm{Sn}^{2+}$ 结合. 通过 $\mathrm{Sn}^{2+}$ 在乙醇-水介质中的水解作用得到核-壳复合结构, 再经高温怶 烧, 得到 $\mathrm{SnO}_{2}$ 中空微纳米球. 实验对前驱体的浓度、表面活性剂的用量、反应时间及模板选择等方面做了研究, 通过扫描电镜 (SEM)、 $X$ 射线衍射 ( $X R D)$ 、红外 (IR) 光谱、热重分析 (TGA)、 $H_{2}$ 程序升温还原 $\left(H_{2}-T P R\right)$ 、 Brunauer-Emmett-Teller (BET)比表面积等技术深入探究 $\mathrm{SnO}_{2}$ 中空微纳米球的结构, 并对比中空 $\mathrm{SnO}_{2}$ 与实心 粒子的氧化还原特性. BET 和 $\mathrm{H}_{2}-\mathrm{TPR}$ 显示将 $\mathrm{SnO}_{2}$ 制备成微纳米空心球后其比表面积增大, 表面氧空位明显增 多, 氧化活性明显提高. 从 IR及 XRD 推断核-壳结构形成机理, 进而优化出简单合理的实验方案, 获得表面光 滑、结构致密, 包覆厚度可控的 $\mathrm{SnO}_{2}$ 中空微纳米球.
\end{abstract}

关键词: $\mathrm{SnO}_{2}$; 中空微纳米球; 核-壳结构; 模板法; 水解反应 中图分类号: 0643; 0649

\section{Preparation and Properties of Monodisperse $\mathrm{SnO}_{2}$ Hollow Micro/Nano Spheres}

\author{
CAI Hong-Min ${ }^{1}$ \\ REN Su-Zhen ${ }^{1, *}$ \\ WANG Meng ${ }^{1}$ \\ JIA Cui-Ying ${ }^{2}$ \\ ('College of Chemistry, Dalian University of Technology, Dalian 116024, Liaoning Province, P. R. China; \\ ${ }^{2}$ The Experiment Center of Chemistry, Dalian University of Technology, Dalian 116024, Liaoning Province, P. R. China)
}

\begin{abstract}
Templating is one of the most important methods for preparation of inorganic hollow micro/ nano spheres. We prepared monodisperse polystyrene (PS) microspheres having a diameter of $620 \mathrm{~nm}$ by the emulsion polymerization of styrene. Sulfonated polystyrene (PSS) microspheres were used as a template, through electrostatic adsorption of anions and cations, for modification with $\mathrm{Sn}^{2+}$ from $\mathrm{SnSO}_{4}$ precursor. The core-shell composite structures thereby produced through $\mathrm{Sn}^{2+}$ hydrolysis in an ethanolwater medium were calcined at high temperature to remove PSS and to obtain $\mathrm{SnO}_{2}$ hollow micro/nano spheres. We investigated the effects of precursor concentration, amount of surfactant, reaction time, and templates choice. Scanning electron microscopy (SEM), X-ray diffraction (XRD), infrared (IR) spectroscopy, thermogravimetric analysis (TGA), $H_{2}$ temperature programmed reduction ( $\left.H_{2}-T P R\right)$, Brunauer-Emmett-Teller (BET) measurement, and other technical probes were used to detect the structure and properties of the prepared $\mathrm{SnO}_{2}$ hollow micro/nano spheres, and compared them with those of solid $\mathrm{SnO}_{2}$. BET and $\mathrm{H}_{2}$-TPR showed that the hollow $\mathrm{SnO}_{2}$ micro/nano spheres had improved specific surface area, surface oxygen vacancies, and oxidation activity. We inferred the growth mechanism of the core-shell structure from IR spectroscopy and XRD pattern and optimized the simple and reasonable synthesis procedure to obtain $\mathrm{SnO}_{2}$ hollow micro/nano spheres which had smooth surface, compact structure, and well controlled cladding thickness.
\end{abstract}

Received: November 26, 2012; Revised: January 30, 2013; Published on Web: February 5, 2013.

"Corresponding author. Email: rensz@dlut.edu.cn; Tel: +86-411-84986074.

The project was supported by the National Natural Science Foundation of China (21176043).

国家自然科学基金(21176043)资助项目

(C) Editorial office of Acta Physico-Chimica Sinica 
Key Words: $\mathrm{SnO}_{2} ; \quad$ Hollow micro/nano sphere; Core-shell structure; Template method; Hydrolysis

\section{1 引言}

具有特殊结构的纳米材料一直是人们研究的 热点. 纳米复合微球及中空微纳米球具有密度低、 比表面积大、催化活性高等特点, 被广泛应用到药 物载体封装、电极材料、污水处理、催化等方面..$^{1-8}$ 在 生物、化学、材料等领域激发了人们极大的研究热 情.

$\mathrm{SnO}_{2}$ 是典型的宽带隙、 $n$ 型半导体材料 $(E=3.6$ $\mathrm{eV})$. 不同形貌的 $\mathrm{SnO}_{2}$ 晶体, 具有不同的物理、化学 及电化学行为. 其中空材料的高渗透性、高缓冲性 能、高可塑性, 使其合成研究备受关注. Xiao 等 ${ }^{9}$ 在 乙醇酸性介质中, 不添加任何模板直接获得 $\mathrm{SnO}_{2}$ 中 空球. Zhao 等 ${ }^{10}$ 利用十二烷基苯磺酸钠(SDBS)为软 模板获得 $\mathrm{SnO}_{2}$ 中空结构. 但究其形貌, 这些中空球 的分散性、粒径的均一性及可控性达不到理想效 果. 如何获得均一稳定的中空微纳米球将是本文研 究的重点.

硬模板法是获得特定形貌和尺寸纳米材料的 有效方法. 通过不同模板的控制、剪裁, 可得到理想 的纳米应用材料. 如无机物模板: $\mathrm{SiO}_{2} @$ 杂化贵金 属、 ${ }^{11} \mathrm{SiO}_{2} @ \mathrm{Ag} 、{ }^{12} \mathrm{NaCl} @ \mathrm{SnO}_{2}{ }^{13}$ 等. 由模板的特定形 貌可分别得到球形、立方中空材料. 高分子聚合物 的形貌可控易得, 常用作核-壳结构的模板PMMA@合金、年 $\mathrm{PS} @ \mathrm{SiO}_{2} 、{ }^{15} \mathrm{PS} @ \mathrm{TiO}_{2}{ }^{16}$ 等. 利用有 机溶剂溶解或者高温煅烧等手段将模板去除, 便得 到中空微纳米结构.

$\mathrm{PS}$ 的玻璃化转变温度相对较低, 与 $\mathrm{SnO}_{2}$ 高温成 相矛盾, 在室温下合成 $\mathrm{PS} @ \mathrm{SnO}_{2}$ 的报道并不多. $\mathrm{Li}$ 等 ${ }^{17}$ 以叔丁氧基锡为前驱体在室温下得到 $\mathrm{PS} @ \mathrm{SnO}_{2}$ 复合结构, 但有机锡化物的成本相对较高, 如何利 用无机锡盐得到 PS@ $\mathrm{SnO}_{2}$ 复合结构将成为我们研 究的出发点.

我们将 PS 表面进行改性, 其表面负电荷与无机 盐阳离子结合, 在聚合物表面进行包覆, 得到核-壳 微纳米复合结构. 利用高温煅烧, 将有机物模板去 除, 得到晶型良好的 $\mathrm{SnO}_{2}$ 中空微纳米球. 本实验分 别对模板的作用、反应时间、反应浓度、核-壳结构及 中空球的性质予以研究. 优化出简单、可控、条件温 和的微纳米复合结构的合成方法. 最终得到均一、 稳定、高度分散的中空微纳米球. 通过此合成思路
我们将拓展合金核-壳复合结构的研究.

\section{2 实验部分}

\section{1 主要原料}

苯乙烯 $(\mathrm{St})$, 化学纯, 国药集团化学试剂有限公 司; 过硫酸钾(KPS), 分析纯, 用去离子水重结晶后 使用; 氢氧化钠 $(\mathrm{NaOH})$, 分析纯; 无水乙醇 $(\mathrm{EtOH})$, 分析纯; 浓硫酸 $\left(\mathrm{H}_{2} \mathrm{SO}_{4}, 98 \%\right)$, 分析纯; $\mathrm{SnSO}_{4}$, 分析 纯; 聚乙烯吡咯烷酮 $\left(\mathrm{PVP}, M_{\mathrm{w}}=40000\right)$, 分析纯; 所用 水均为去离子水.

\section{2 单分散聚苯乙烯微球的制备}

\subsection{1 苯乙烯的预处理}

用 $200 \mathrm{~mL}, 0.1 \mathrm{~mol} \cdot \mathrm{L}^{-1} \mathrm{NaOH}$ 分四次洗涤 St. 可 观察到体系上下界面分层, 上层为无色 $\mathrm{St}$, 下层为 淡淡的粉红色 $\mathrm{NaOH}$ 溶液. 用去离子水分别洗涤 $4-5$ 次, 至体系 $\mathrm{pH}=7$.

\section{2 .2 单分散 PS 微球的制备}

用无㿝乳液聚合法制备单分散 PS 模板微球. ${ }^{18}$ 并予以适当改进: 分别将 $300 \mathrm{~mL}$ 去离子水、 $40 \mathrm{~mL}$ 乙醇、 $40 \mathrm{~mL} \mathrm{St}$ 注入 $500 \mathrm{~mL}$ 三口圆底烧瓶中. 水热 $70{ }^{\circ} \mathrm{C}$, 通入 $\mathrm{N}_{2}$, 大约 $30 \mathrm{~min}$ 后加 $20 \mathrm{~mL} \mathrm{KPS}(0.1326$ $\mathrm{g}$ )水溶液. 反应时间 $24 \mathrm{~h}$. 冷却至室温后, 将所得乳 液超声分散 $1 \mathrm{~h}$, 在 $2500 \mathrm{r} \cdot \mathrm{min}^{-1}$ 下离心 $4 \mathrm{~h}$. 所得产 物真空条件下, $60^{\circ} \mathrm{C}$ 干燥过夜, 得到白色粉末.

\section{3 磺化聚苯乙烯微球(PSS)的制备}

利用 $98 \%$ 的浓硫酸直接磺化合成的 PS 微球. ${ }^{19}$ 具体方法: 将 $1 \mathrm{~g}$ 干燥后的 PS 粉末分散至含有 30 $\mathrm{mL}$ 的浓硫酸 $\left(\mathrm{H}_{2} \mathrm{SO}_{4}\right.$ 体积分数为 $\left.98 \%\right)$ 的圆底烧瓶 中. 超声 $30 \mathrm{~min}$ 后, 在 $40{ }^{\circ} \mathrm{C}$ 水浴中搅拌 $4 \mathrm{~h}$. 反应结 束后用去离子水将体系稀释, 在 $5000 \mathrm{r} \cdot \mathrm{min}^{-1}$ 下离心 $20 \mathrm{~min}$, 除去上清液, 用去离子水洗涤多次至中性. 产物真空条件下, $60^{\circ} \mathrm{C}$ 干燥过夜, 得到白色粉末.

\section{4 核壳结构PSS@SnO 2 复合微球的制备}

将 $0.05 \mathrm{~g}$ PSS 分散到含有 $10 \mathrm{~mL}$ 无水乙醇的圆 底烧瓶中, 超声 $10 \mathrm{~min}$ 后, 加入 $10 \mathrm{~mL}$ PVP $(0.025 \mathrm{~g})$ 水溶液, $25^{\circ} \mathrm{C}$, 摚拌 $10 \mathrm{~min}$, 再缓慢滴加 $10 \mathrm{~mL}$ $\mathrm{SnSO}_{4}\left(0.05 \mathrm{~mol} \cdot \mathrm{L}^{-1}\right)$ 水溶液, 体系成乳白色, 反应 $12 \mathrm{~h}$ 后停止. 将产物在 $3000 \mathrm{r} \cdot \mathrm{min}^{-1}$ 下离心 $5 \mathrm{~min}$, 除 去上清液, 用乙醇和去离子水分别洗涤多次至中 性. 将离心后产物在真空条件下于 $60^{\circ} \mathrm{C}$ 干燥过夜, 


\section{得到白色粉末.}

\section{$2.5 \mathrm{SnO}_{2}$ 中空微球的制备}

将 2.4 节中所得核-壳结构 PSS@ $\mathrm{SnO}_{2}$ 复合微球 置于马弗炉中, 以 $2^{\circ} \mathrm{C} \cdot \mathrm{min}^{-1}$ 的速率, 从室温升至 $550{ }^{\circ} \mathrm{C}$, 煅烧 $2 \mathrm{~h}$ 后得到灰白色粉末.

\section{6 表 征}

扫描电镜 $(S E M)$ 观察采用美国 FEI 公司 QUANTA 450 高分辨电镜, 观察微球形貌. $\mathrm{X}$ 射线衍射 (XRD) 分析在 XD-3A 型 X 射线衍射仪上进行. 测试 采用 $\mathrm{Cu} K_{a}$ 射线源 $(\lambda=0.15418 \mathrm{~nm})$, 工作电压 $40 \mathrm{kV}$, 电流 $100 \mathrm{~mA}$, 扫描速率是 $10\left(^{\circ}\right) \cdot \mathrm{min}^{-1}$, 扫描范围 $20^{\circ}-80^{\circ}$. 红外(IR)光谱在德国布鲁克光谱仪器公司 的 Bruker-Tenson 27 型红外光谱仪进行分析. 热重分 析(TGA) 在美国 TA 公司的Q50 型热重分析仪进行. $\mathrm{N}_{2}$ 条件下由室温升温至 $800{ }^{\circ} \mathrm{C}$ (速率为 $10^{\circ} \mathrm{C}$. $\mathrm{min}^{-1}$ ). 比表面测定(BET)在北京精微高博公司 JWBK122W 型的比表面及孔径测定仪上进行. $\mathrm{H}_{2}$ 程序 升温还原 $\left(\mathrm{H}_{2}-\mathrm{TPR}\right)$ 测试在美国康塔公司 CHEMBET TPD/TPR 型的程序升温化学吸附仪上进行. 每个样 品 $(0.1 \mathrm{~g})$ 在 $\mathrm{He}$ 气 $\left(100 \mathrm{~mL} \cdot \mathrm{min}^{-1}\right)$ 下吹扫, $550{ }^{\circ} \mathrm{C}$ 保持 $30 \mathrm{~min}$, 冷却到 $150^{\circ} \mathrm{C}$. 在反应气 $\mathrm{H}_{2}\left(100 \mathrm{~mL} \cdot \mathrm{min}^{-1}\right)$ 以 $10{ }^{\circ} \mathrm{C} \cdot \mathrm{min}^{-1}$ 的速率由 $150{ }^{\circ} \mathrm{C}$ 升温至 $800^{\circ} \mathrm{C}$.

\section{3 结果与讨论}

\section{1 单分散的PS 及 PSS 微球}

无皇乳液聚合法制备的单分散 PS 微球(见图 1 (a))大小均一, 数均粒径为 $620 \mathrm{~nm}$. 从 SEM 图中可得 到 PS 的粒径分布情况. 通过对 PS 进行磺化反应, 得 到单分散的 PSS 模板微球(见图 1(b)), 微球大小均 一, 粒径尺寸无明显变化.

\section{2 核-壳结构PSS@ $\mathrm{SnO}_{2}$ 复合微球及 $\mathrm{SnO}_{2}$ 中 空球形貌}

3.2.1 反应时间对核-壳结构PSS@ $\mathrm{SnO}_{2}$ 复合 微球及 $\mathrm{SnO}_{2}$ 中空球形貌的影响

表 1 列出了反应时间对核-壳结构形貌的影响,
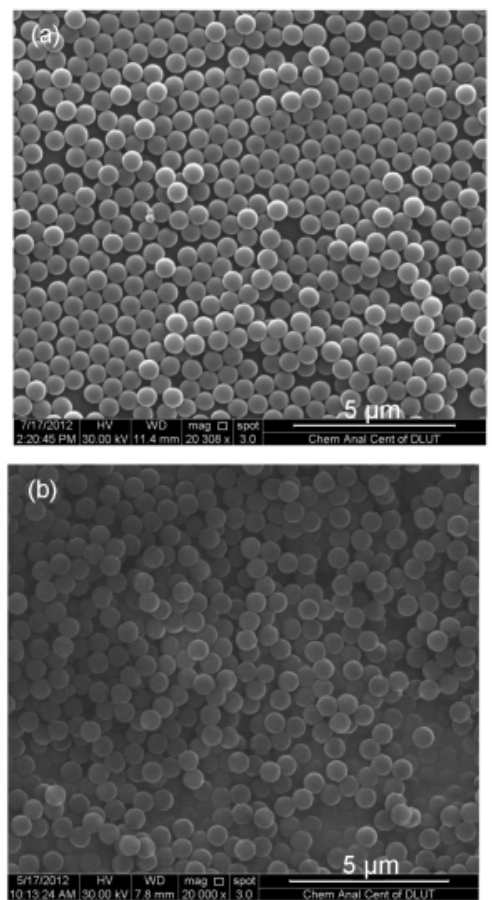

图 1 PS (a)与 PSS (b) 的 SEM 图

Fig.1 SEM images of PS (a) and PSS (b)

PS: polystyrene; PSS: sulfonated polystyrene

图 2 是不同反应时间的样品经煅烧后的 SEM 图. 从 表 1 和图 2 可观察到不同反应时间对 PSS@ $\mathrm{SnO}_{2}$ 核壳结构形貌的影响. 在相同的配比和介质条件下, 控制滴加后的反应时间. 分别为 $10 \mathrm{~min} 、 30 \mathrm{~min} 、 2$ $h 、 12 h 、 14 h($ 表 1$)$. 其中样品 $a 、 b$ 反应干燥后为黄色 粉末, 样品 $c 、 d 、 e$ 反应干燥后为白色粉末. 由样品 $a-e$ 干燥煅烧后的 SEM 图(图 2)可得到如下结论: 反 应 10、30 min 时, 为不完全包覆, 从图 2(a,b)可猜想, 在反应初始前驱体阳离子迅速与模板上的 $\mathrm{SO}_{3} \mathrm{H}-$ 结合形成包覆, 但反应时间较短包覆层并不完全, 煅烧后随模板的燃烧薄壳层坍塌, 只剩下坞塌后的 碎片. 随反应时间延长, 模板逐渐被包覆. 在图 2(b) 中可观察到模板燃烧后的球形趋势. 当反应延长至 $2 \mathrm{~h}$ 时可观察到球壳完全包覆, 但壳壁较薄, 煅烧后 有很多破裂现象. 继续延长反应时间, 当反应时间 为 $12 \mathrm{~h}$ 时, 得到煅烧后的图 2(c). 从图中可得到表面

表 1 反应时间对核-壳结构形貌的影响

Table1 Influence of reaction time on core-shell structure morphology

\begin{tabular}{ccccccc}
\hline Sample & Template & $c\left(\mathrm{SnSO}_{4}\right) /\left(\mathrm{mol}^{\circ} \mathrm{L}^{-1}\right)$ & $m(\mathrm{PVP}) / \mathrm{g}$ & Reaction time/h & Shell morphology & Shell morphology after calcination \\
\hline $\mathrm{a}$ & PSS & 0.05 & 0.025 & $1 / 6$ & partly & partly shell \\
$\mathrm{b}$ & PSS & 0.05 & 0.025 & $1 / 2$ & partly & partly shell \\
$\mathrm{c}$ & PSS & 0.05 & 0.025 & 2 & whole & hollow \\
$\mathrm{d}$ & PSS & 0.05 & 0.025 & 12 & whole & hollow \\
$\mathrm{e}$ & PSS & 0.05 & 0.025 & 14 & whole & hollow (the second particles) \\
\hline
\end{tabular}



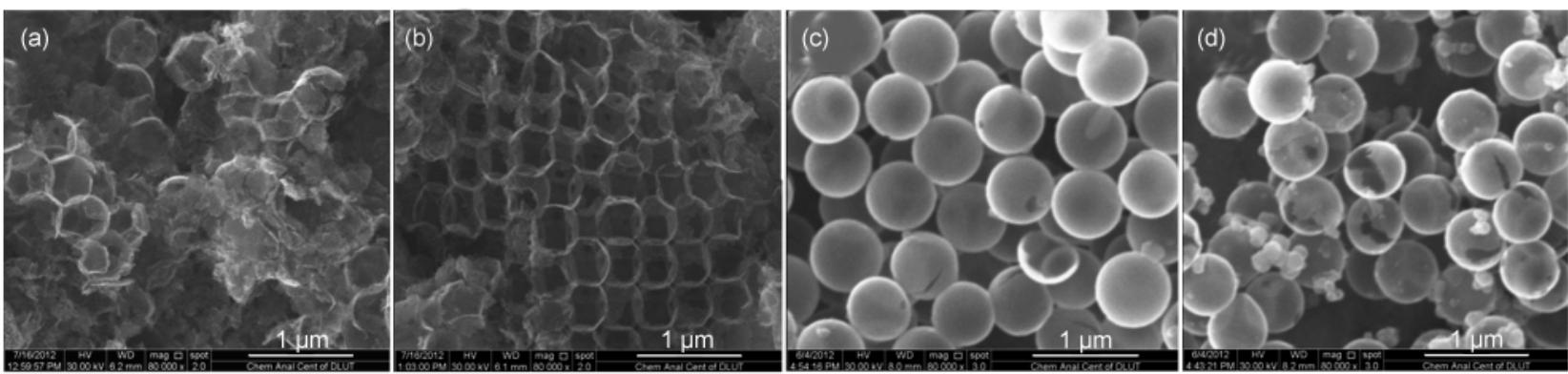

图 2 不同反应时间的样品经煅烧后的 SEM 图

Fig.2 SEM images of samples prepared with different reaction time after calcination

(a) $10 \mathrm{~min}$, (b) $30 \mathrm{~min}$, (c) $12 \mathrm{~h}$, (d) $14 \mathrm{~h}$

光滑、粒径均一、单分散的微球结构. 当反应延长至 $14 \mathrm{~h}$ 时, 由图 2(d) 可观察到在得到单分散的微球结 构同时, 出现次级粒子, 相互团聚在一起. 综上, 我 们得到前驱体 $0.05 \mathrm{~mol} \cdot \mathrm{L}^{-1}$, PVP $0.025 \mathrm{~g}$ 的配比下, 最佳反应时间为 $12 \mathrm{~h}$.

图 3 给出了煅烧前后样品的 SEM 图. 通过 $\operatorname{SEM}$ (未喷金处理)炷烧前后的对比可近一步证实核 壳结构向中空球的转化. 从图中对比可发现, 在相

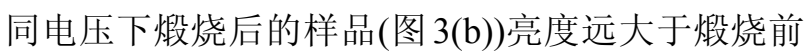
的样品(图 3(a)). 煅烧前样品均粒径为 $670 \mathrm{~nm}$, 衫烧 后样品均粒径为 $650 \mathrm{~nm}$. 㷽烧后的样品均粒径减少 了 $20 \mathrm{~nm}$ 左右, 这是由于高温衫烧过程, 随聚合物模 板的燃烧, 样品发生萎缩现象. 从图 3(b)的半球壳还 有煅烧后的裂痕可证实㷽烧后得到中空球结构(右 上角图: 相同样品在 $550{ }^{\circ} \mathrm{C}$ 煅烧 $2 \mathrm{~h}, 5^{\circ} \mathrm{C} \cdot \mathrm{min}^{-1}$ 煅
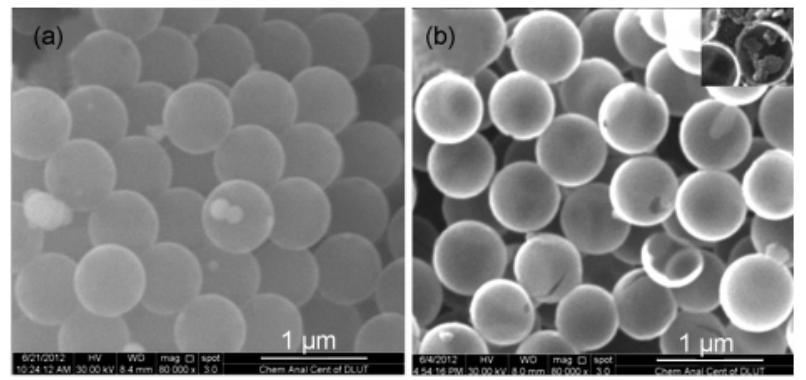

图 3 煅烧前(a)后(b)样品的SEM图

Fig.3 SEM images of samples before (a) and after (b) calcination

reaction time: $12 \mathrm{~h}, m(\mathrm{PVP})=0.025 \mathrm{~g}$; inset in figure (b) shows the hollow spheres of the sample after calcination $\left(550{ }^{\circ} \mathrm{C}, 2 \mathrm{~h}, 5^{\circ} \mathrm{C} \cdot \mathrm{min}^{-1}\right)$.
烧条件下得到的破碎球壳).

3.2.2 表面活性剂对核壳结构PSS@SnO 2 复合 微球及 $\mathrm{SnO}_{2}$ 中空球形貌的影响

PVP 作为一种化学性质稳定的非离子表面活 性剂, 与其他化合物有良好的螯合能力, 被广泛应 用到纳米粒子的制备中. ${ }^{20}$ 表 2 列出了不同量 PVP对 核-壳结构形貌的影响, 图 4 为含不同量 PVP 的样品 煅烧后的 SEM 图. 通过表 2 与图 4 分别对不同量的 PVP 参与反应进行对比. 首先, 在相同反应时间, 将 添加与不添加 PVP 做对比. 从图 4(a)、4(b) 中可以看 出, 虽然两个样品敖烧后均无中空球结构, 但添加 $\operatorname{PVP}($ 图 4(b)) 明显有助于球壳结构的形成. 在 $f 、 a$ 样 对比的基础上延长反应时间至 $2 \mathrm{~h}$ 以上, 我们发现 煅烧后均可形成中空球结构, 粒径大小均一程度差 别不大. 其次, 我们对煅烧前后的样品进行对比(在 相同反应时间 $12 \mathrm{~h}$, 将 PVP 的质量增加一倍). 对比 图 3(b)和图 4(c) 可以看出, 增加 PVP 的量可明显改 变球壳的粗粘度, 同时有少量次级粒子生成. 这是 由于 PVP 作为双亲分子, 能在模板表面有序排列, 在模板与前驱体之间起到一定的交联作用, 有助于 球形包覆的形成. 同时 PVP 在模板表面形成负电 荷 ${ }^{21}$ 与 $\mathrm{Sn}^{2+}$ 发生静电作用. 但这两种作用力相对较 弱, 在一定时间范围内有无 PVP 的添加对实验结果 影响不大. 但当 PVP 浓度增大时, 较长的有机链易 形成网络结构, 对粒子产生多层包裹作用, 促使次 级粒子的大量产生. 所以少量的 PVP 添加有助于壳 层结构的包覆, 但过量的 PVP 则导致壳层表面粗

表 2 不同量 PVP 对核-壳结构形貌的影响

Table 2 Influence of different amount PVP on core-shell structure morphology

\begin{tabular}{ccccccc}
\hline Sample & Template & $c\left(\mathrm{SnSO}_{4}\right) /\left(\mathrm{mol} \cdot \mathrm{L}^{-1}\right)$ & $m(\mathrm{PVP}) / \mathrm{g}$ & Reaction time $/ \mathrm{h}$ & Shell morphology & Shell morphology after calcination \\
\hline $\mathrm{f}$ & PSS & 0.05 & 0 & $1 / 6$ & partly & no shell \\
$\mathrm{a}$ & PSS & 0.05 & 0.025 & $1 / 6$ & partly & partly shell \\
$\mathrm{d}$ & PSS & 0.05 & 0.025 & 12 & whole & hollow (smooth surface) \\
$\mathrm{g}$ & PSS & 0.05 & 0.05 & 12 & whole & hollow (coarse surface) \\
\hline
\end{tabular}



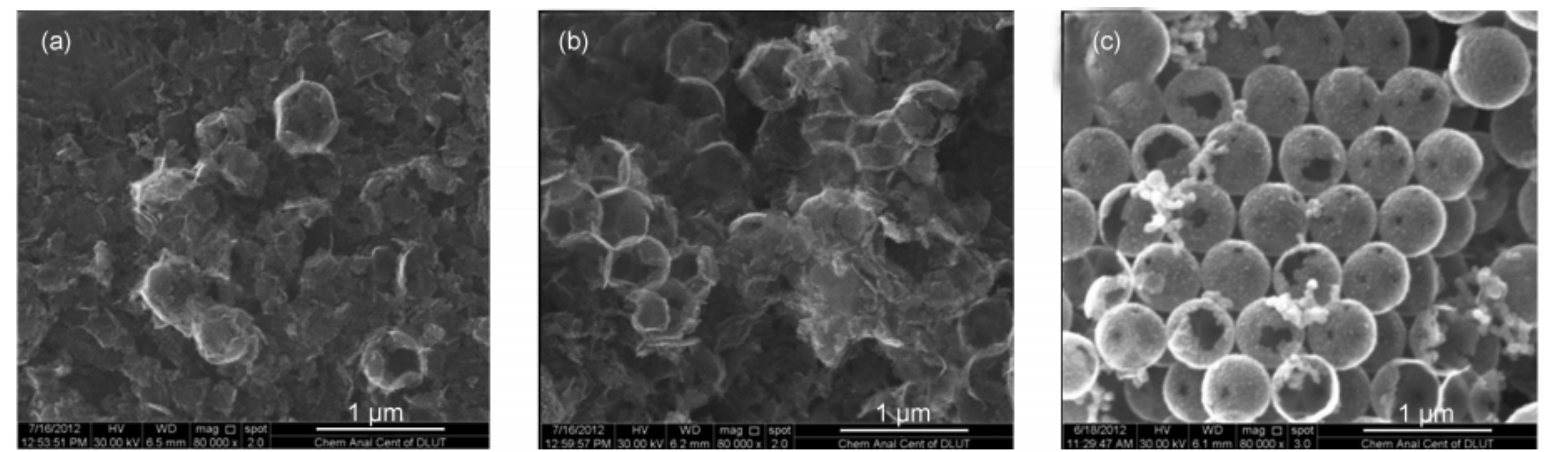

图 4 含不同量 PVP 的样品煅烧后的 SEM 图

Fig.4 SEM images of samples with different amount of PVP after calcination

(a) $0 \mathrm{~g}$ (the reaction time is $10 \mathrm{~min}$ ), (b) $0.025 \mathrm{~g}$ (the reaction time is $10 \mathrm{~min}$ ), (c) $0.05 \mathrm{~g}$ (the reaction time is $12 \mathrm{~h}$ )

䊁, 次级粒子生成.

3.2.3 不同前驱体浓度对核壳PSS@SnO 2 复合微 球及 $\mathrm{SnO}_{2}$ 中空球形貌的影响

表 3 介绍了前驱体 $\left(\mathrm{SnSO}_{4}\right)$ 浓度对核-壳结构的 影响, 将产物煅烧后得到其 SEM 图(图 5). 在表 3 和 图 5 中, 分别对 $0.05 、 0.1 、 0.2 \mathrm{~mol} \cdot \mathrm{L}^{-1}$ 的 $\mathrm{SnSO}_{4}$ 在 $12 \mathrm{~h}$ 的反应条件下进行比较. 随前驱体浓度的增大, 煅 烧后中空球的直径随之增大. 随着煅烧模板的去除 粒径会相应缩小. 在共同作用下, 均粒径分别为 650、680、690 nm. 且随着前驱体浓度的增加反应开 始出现次级粒子, 当浓度达到 $0.2 \mathrm{~mol} \cdot \mathrm{L}^{-1}$ 时, 次级粒 子为 150-200 nm (煅烧前与煅烧后直径对比). 要得 到表面均一稳定的中空球结构, 控制反应物的浓度 是十分必要的.

\subsection{4 模板对核壳结构PSS@ $\mathrm{SnO}_{2}$ 复合微球及 $\mathrm{SnO}_{2}$ 中空球形貌的影响}

通过以上实验 PSS 作为改性的聚合物模板对核 壳复合结构和中空球的获得起到了决定性作用. 下 面以未改性的聚合物 PS 为模板与无聚合物模板的 样品进行对比实验 (表 4). 图 6(a,b) 为以 PS 为模板, 不经磺化直接进行反应. 从图 6(a)可看到煅烧前体 系有均一的大球和不均一的次级粒子共同存在. 次 级粒子的尺寸不均, 大约在 150-200 nm 之间. 煅烧 后(图 6(b))大球全部消失, 只剩下大小不均的次级粒 子. 图 6(a,b) 说明未改性的 PS 不能作为模板形成核 壳结构, 其表面的作用力与前驱体相互作用力不足 以达到包覆效果. 图 6(c, d) 是未添加模板条件下, 将 前驱体在 PVP 存在的介质条件下进行反应, 得到

表 3 前驱体 $\left(\mathrm{SnSO}_{4}\right)$ 浓度对核-壳结构的影响

Table 3 Influence of the concentration of precursor $\left(\mathrm{SnSO}_{4}\right)$ on core-shell structure morphology

\begin{tabular}{ccccccc}
\hline Sample & Template & $c\left(\mathrm{SnSO}_{4}\right) /\left(\mathrm{mol} \cdot \mathrm{L}^{-1}\right)$ & $m(\mathrm{PVP}) / \mathrm{g}$ & Reaction time/h & Shell morphology & Shell morphology after calcination \\
\hline $\mathrm{d}$ & PSS & 0.05 & 0.025 & 12 & whole & hollow (smooth surface) \\
h & PSS & 0.1 & 0.025 & 12 & whole & hollow (much thick shell, the second particles) \\
i & PSS & 0.2 & 0.025 & 12 & whole & hollow (coarse surface, the second particles) \\
\hline
\end{tabular}
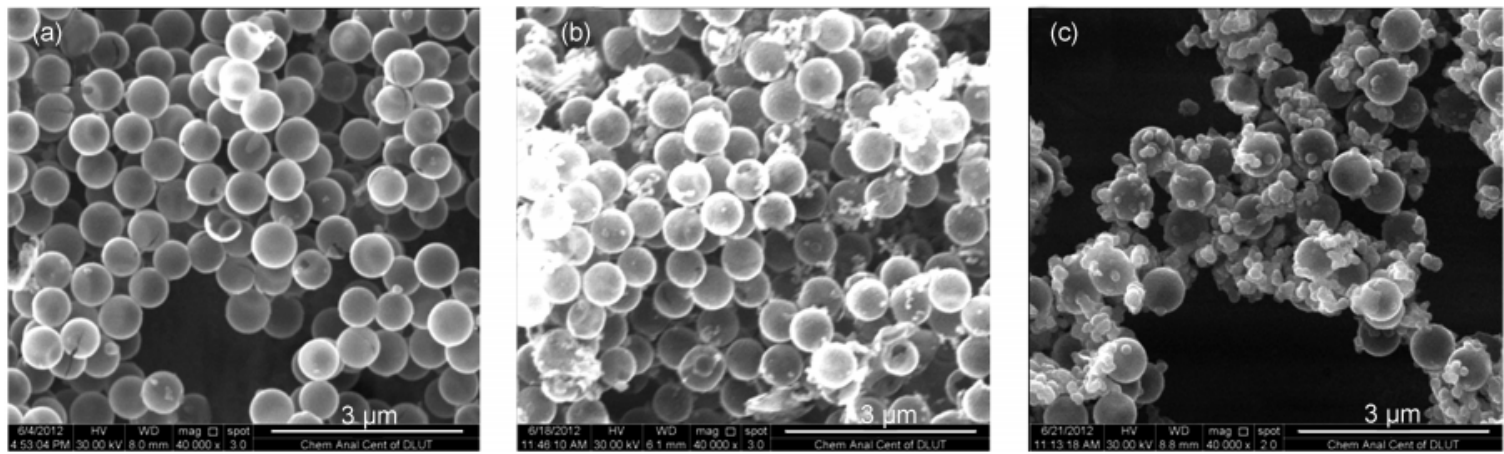

图 5 不同浓度的前驱体煅烧后的 SEM图

Fig.5 SEM images of precursor with different concentrations after calcination $c\left(\mathrm{SnSO}_{4}\right) /\left(\mathrm{mol} \cdot \mathrm{L}^{-1}\right):$ (a) 0.05 , (b) 0.1 , (c) 0.2 
表4 不同模板对核-壳结构形貌的影响

Table 4 Influence of the different templates on core-shell structure morphology

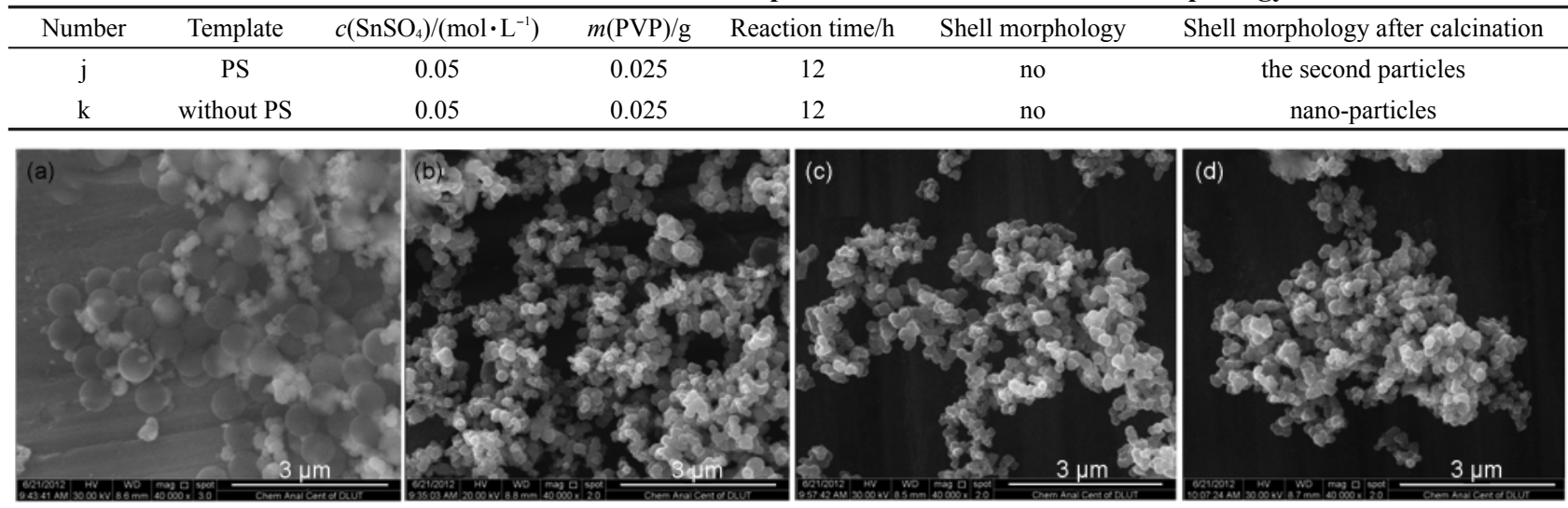

图 6 有 $(a, b)$ 无 $(c, d)$ 模板时样品煅烧前 $(a, c)$ 后 $(b, d)$ 的 SEM 图

Fig.6 SEM images of samples with $(a, b)$ or without $(c, d)$ template before $(a, c)$ and after $(b, d)$ calcination

200-250 nm 的纳米粒子. 从实验可知, 将 PS 模板改 性是核壳结构形成的关键因素. 正是改性聚合物表 面和前驱体的相互作用使得核壳结构的粒径可控, 炦烧后得到均一稳定的微纳米空心球.

\section{3 结构、性质表征及机理推断}

\subsection{1 结构及机理推断}

$\mathrm{SnO}_{2}$ 中空球的晶体结构通过 XRD 谱图(图 7)获 得, 未殷烧的PSS@ $\mathrm{SnO}_{2}$ 核壳结构无明显衍射峰(图 7(a)), 属无定向状态. 绠烧后的中空球有明显的衍射 峰(图 7(b, c, d)), 吸收峰与四晶相金红石 $\mathrm{SnO}_{2}$ 相一 致. 从而得到, 煅烧是 $\mathrm{SnO}_{2}$ 晶型结构形成的决速步 骤, 并且随着炦烧温度的提高, 衍射峰逐渐增强, 呈 现更好的晶型与更大的晶体尺寸. 但从 SEM 图观 察, 经 $750{ }^{\circ} \mathrm{C}$ 高温陆烧后中空球破碎, 得不到表面 均一光滑的中空形貌. 㷽烧温度要小于 $750{ }^{\circ} \mathrm{C}$. 由 TGA 图(图 8)可知, 改性后的 PSS 模板热稳定性提 高, 分解温度为 $360-480{ }^{\circ} \mathrm{C}$. 不考虑水的失重,

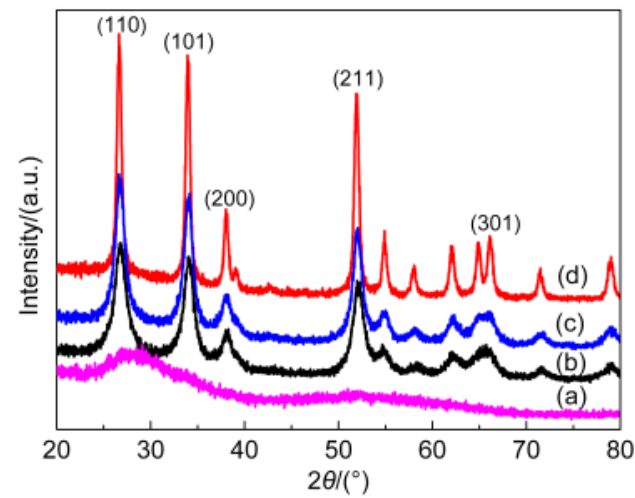

图 $7 \mathrm{PSS} @ \mathrm{SnO}_{2}$ 核-壳微球煅烧前 $(\mathrm{a})$ 后 $(\mathrm{b}, \mathrm{c}, \mathrm{d})$ 的 XRD 图 Fig.7 XRD patterns of PSS@SnO $\mathrm{SnO}_{2}$ core-shell microspheres before (a) and after (b, c, d) calcination calcination temperature: (b) $550{ }^{\circ} \mathrm{C}$, (c) $650{ }^{\circ} \mathrm{C}$, (d) $750{ }^{\circ} \mathrm{C}$
$\mathrm{PSS} @ \mathrm{SnO}_{2}$ 在 $360-480{ }^{\circ} \mathrm{C}$ 的质量损失约为 $39 \%$. 为 了获得理想的晶型及中空形貌, 我们将煅烧温度控 制在 $550^{\circ} \mathrm{C}$, 煅烧 $2 \mathrm{~h}$.

图 9 为 $\mathrm{PS} 、 \mathrm{PSS} @ \mathrm{SnO}_{2} 、 \mathrm{SnO}_{2}$ 红外光谱图比较. 由 PS 的 IR 谱图(图 9(a)) 可知, 1450、1470 和 1590 $\mathrm{cm}^{-1}$ 三个中等强度的吸收带对应于苯环的 $\mathrm{C}=\mathrm{C}$ 伸 缩振动. $700 \mathrm{~cm}^{-1}$ 处的吸收峰对应于芳香骨架面外 弯曲振动, 而 $750 \mathrm{~cm}^{-1}$ 处的吸收峰对应于苯环 $=\mathrm{C}-$ $\mathrm{H}$ 的面外弯曲振动. 这两个区内同时出现强吸收峰 说明苯环上有 5 个相邻氢的苯核(苯环为单取代), 这 与 2000-1660 $\mathrm{cm}^{-1}$ 区内四个吸收带一致. ${ }^{22} \mathrm{PSS} / \mathrm{SnO}_{2}$ 与 PS 的 IR 谱图比较(图 9(b)), 在 $900 \mathrm{~cm}^{-1}$ 出现苯环 $=\mathrm{C}-\mathrm{H}$ 面外弯曲振动峰, 由单取代转为 1,3 取代. 同时, 在 $1050 \mathrm{~cm}^{-1}$ 处出现磺酸 $\mathrm{S}=\mathrm{O}$ 对称伸缩振动 峰, 1370-1335 $\mathrm{cm}^{-1}$ (双峰)、 $1170 \mathrm{~cm}^{-1}$ 处分别出现磺 酸酯 $\mathrm{S}=\mathrm{O}$ 不对称与对称伸缩振动峰, 表明磺化 $\mathrm{PS}$ 的形成. PSS 亲水性增强, 在 $3400 \mathrm{~cm}^{-1}$ 处呈现越来

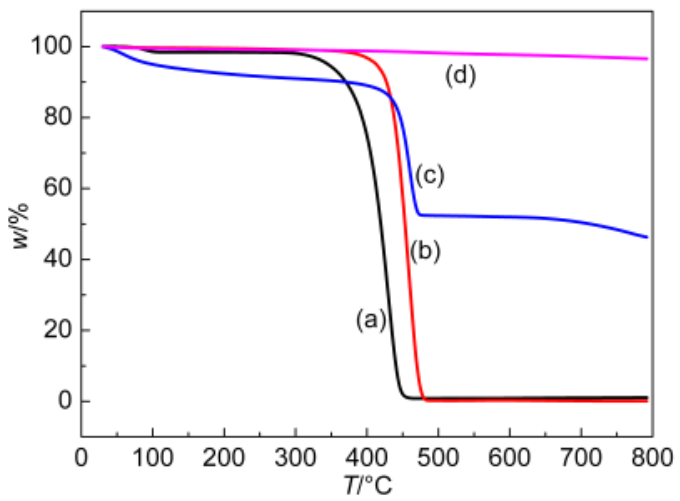

图 8 样品热重分析(TGA)谱图

Fig.8 Thermogravimetric analysis (TGA) trace of samples (a) PS, (b) PSS, (c) PSS@ $\mathrm{SnO}_{2}$ (before calcination),

(d) $\mathrm{SnO}_{2}$ (after calcination) 


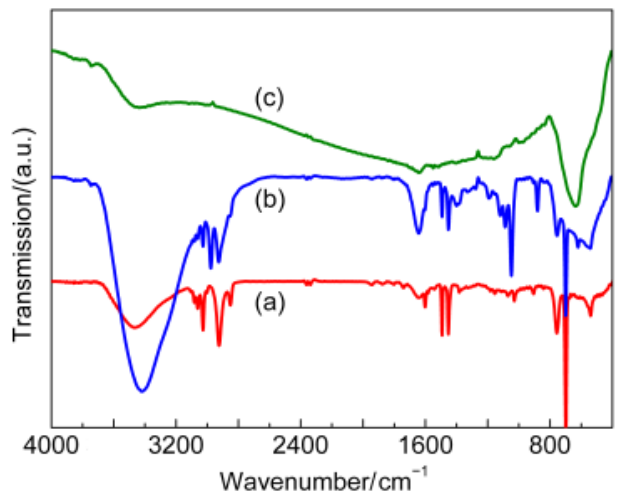

图 9 样品的 IR 谱图

Fig.9 IR spectra of samples

(a) PS, (b) PSS@ $\mathrm{SnO}_{2}$, (c) $\mathrm{SnO}_{2}$

越强的吸收峰. 水分子通过氢键和磺酸基团的氧原 子相互作用, 使一 $\mathrm{OH}$ 基团的伸缩振动吸收峰向低 频方向位移, 谱带加宽, 吸收强度加大.$^{23}$ 与 PSS 相应 的 1600-1450 $\mathrm{cm}^{-1}, 700-750 \mathrm{~cm}^{-1}$ 区域的吸收峰相 对减弱, 说明 $\mathrm{SnO}_{2}$ 包覆在 PSS 表面, 形成核壳结构的 微球. 在图 9(b)中, 400-700 $\mathrm{cm}^{-1}$ 出现 $\mathrm{Sn}-\mathrm{O}$ 及 $\mathrm{Sn}-$ $\mathrm{O}-\mathrm{Sn}$ 吸收振动峰, 在 3100-3700 $\mathrm{cm}^{-1}$ 出现 $\mathrm{Sn}-\mathrm{OH}$ 中 $\mathrm{O}-\mathrm{H}$ 的伸缩振动的宽谱带峰, 更进一步说明 $\mathrm{SnO}_{2}$ 已引入到 PSS 表面. ${ }^{24}$ 经高温煅烧, PSS 特征峰 完全消失, 得到 $\mathrm{SnO}_{2}$ 的红外谱图(图 9(c)), 说明核被 完全去除.

从 IR 与 XRD 谱图我们推断可能的机理: 复合 $\mathrm{PSS} @ \mathrm{SnO}_{2}$ 的形成是由于模板磺酸基负离子与前驱 体 $\mathrm{Sn}^{2+}$ 的静电作用. 无机前驱体被吸附在模板表面, 在乙醇-水介质中被氧化、水解, 反应如下发生:

$2 \mathrm{SnSO}_{4}+\mathrm{O}_{2}+2 \mathrm{H}_{2} \mathrm{O} \rightarrow 2 \mathrm{SnO}_{2}+2 \mathrm{H}_{2} \mathrm{SO}_{4}$

但此时并没有生成晶相的 $\mathrm{SnO}_{2}$, 经高温㷽烧得到晶 相金红石 $\mathrm{SnO}_{2}$.

\subsection{2 氧化还原性质}

由 $\mathrm{H}_{2}$-TPR 谱图(图 10)我们分别得到中空 $\mathrm{SnO}_{2}$ (d 样品煅烧后)及实心 $\mathrm{SnO}_{2}(\mathrm{k}$ 样品煅烧后) 氧化还 原能力的信息. 通过比较两种物质的表面吸附氧、 表面晶格氧和体相晶格氧的活动性能及稳定性能, 得到其氧化还原性能.

$\mathrm{SnO}_{2}$ 在 $\mathrm{H}_{2}$-TPR 中还原 反应有两个过程. ${ }^{25}$ (1) $\mathrm{SnO}_{2}$ 的脱附 $\mathrm{O}^{-}$反应, (2) $\mathrm{SnO}_{2}$ 晶格氧的还原反应(分 别为表面晶格氧与体相晶格氧). 由 TPR 图可知, $\mathrm{SnO}_{2}$ 纳米空心球和纳米粒子在 $250-420{ }^{\circ} \mathrm{C}$ 之间发 生脱附 $\mathrm{O}^{-}$反应, 反应情况几乎完全相同. $420^{\circ} \mathrm{C}$ 以 后, $\mathrm{SnO}_{2}$ 物质开始发生晶格氧的还原反应. 在图中

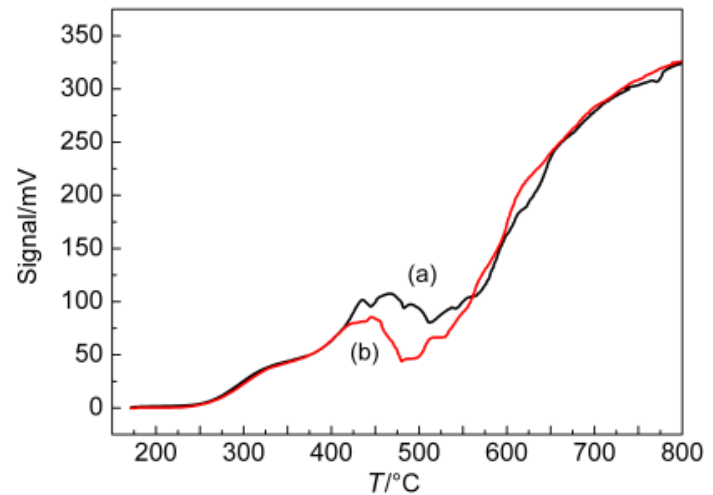

图 10 样品 $\mathrm{H}_{2}$-TPR 分析谱图

Fig.10 $\mathrm{H}_{2}$-TPR trace of samples

(a) hollow $\mathrm{SnO}_{2}$ micro/nano spheres (sample d),

(b) solid $\mathrm{SnO}_{2}$ particles (sample k)

可明显观察到: 在表面晶格氧的反应过程中纳米空 心球的还原强度明显高于实心纳米粒子. 在 $475^{\circ} \mathrm{C}$ 达到最高值(纳米粒子为 $450^{\circ} \mathrm{C}$ ), 于 $510^{\circ} \mathrm{C}$ 结束. 而 实心纳米粒子在 $480^{\circ} \mathrm{C}$ 就提前进入体相晶格氧的 氧化还原反应中. 由此可见, 将 $\mathrm{SnO}_{2}$ 制备成微纳米 空心球后其表面氧空位明显增多, 氧化活性明显提 高. 究其原因, 分别对两种结构进行 $\mathrm{N}_{2}$ 吸附表征, $\mathrm{SnO}_{2}$ 纳米空心球比表面积为 $34.85 \mathrm{~m}^{2} \cdot \mathrm{g}^{-1}$, 平均孔径 $9.39 \mathrm{~nm}$. 而实心纳米粒子只有 $14.52 \mathrm{~m}^{2} \cdot \mathrm{g}^{-1}$. 将 $\mathrm{SnO}_{2}$ 制备成纳米空心球结构, 其大比表面积及多孔特性 是其氧化活性提高的主要原因.

\section{4 结 论}

本文利用无机锡前驱体, 在室温条件下, 简单 方便地制备得到二氧化锡核-壳复合微球. 通过电镜 图可观察到, 对聚合物模板修饰是实现完整包覆的 先提条件, 表面活性剂的添加有助于包覆的形成. 前驱体浓度的确定可调节包覆厚度. 陆烧是形成 $\mathrm{SnO}_{2}$ 金红石晶型的决速步骤. 通过 $\mathrm{H}_{2}$-TPR 测试, 中 空微纳米结构比表面增加, 孔容增大, 表面氧化还 原空位增多, 其氧化活性及催化活性提高, 具有较 好的应用前景. 此外, 通过此合成思路, 我们将进一 步进行合金核-壳结构的制备, 并拓展新型的有机无机杂化囊壳结构.

\section{References}

(1) (a) Zhu, Y.; Shi, J.; Shen, W.; Dong, X.; Feng, J.; Ruan, M.; Li, Y. Angew. Chem. 2005, 117 (32), 5213.

(b) Zhu, Y.; Shi, J.; Shen, W.; Dong, X.; Feng, J.; Ruan, M.; Li, Y. Angew. Chem. Int. Edit. 2005, 44, 5083.

(2) Zhang, W. M.; Hu, J. S.; Guo, Y. G.; Zheng, S. F.; Zhong, L. S.; Song, W. G.; Wan, L. J. Adv. Mater. 2008, 20 (6), 1160. doi: 
10.1002/adma.v20:6

(3) Cai, W. Q.; Yu, J. G.; Cheng, B.; Su, B. L.; Jaroniec, M. J. Phys. Chem. C 2009, 113, 14739. doi: 10.1021/jp904570z

(4) Yu, K.; Wu, Z.; Zhao, Q.; Li, B.; Xie, Y. J. Phys. Chem. C 2008, 112 (7), 2244. doi: 10.1021/jp711880e

(5) Wu, P.; Du, N.; Zhang, H.; Zhai, C. X.; Yang, D. R. ACS Appl. Mater. Interfaces 2011, 3, 1946. doi: 10.1021/am200168w

(6) Zheng, L. R.; Zheng, Y. H.; Chen, C. Q.; Zhan, Y. Y.; Lin, X. Y.; Zheng, Q.; Wei, K. M.; Zhu, J. F. Inorg. Chem. 2009, 48, 1819. doi: 10.1021/ic802293p

(7) Li, W. B.; Bu, Y. Y.; Yu, J. Q. Acta Phys. -Chim. Sin. 2012, 28 (11), 2676. [李卫兵, 补钰显, 于建强. 物理化学学报, 2012, 28 (11), 2676.] doi: 10.3866/PKU.WHXB201207101

(8) Rao, G. S.; Cheng, M. Q.; Zhong, Y.; Deng, X. C.; Yi, F.; Chen, Z. R.; Zhong, Q. L.; Fan, F. R.; Ren, B.; Tian, Z. Q. Acta Phys. -Chim. Sin. 2011, 27 (10), 2373. [ 饶贵仕, 程美琴, 钟 艳, 邓小聪, 易飞, 陈治仁, 钟起玲, 范风茹, 任 斌, 田中群. 物理化学学报, 2011, 27 (10), 2373.] doi: 10.3866/ PKU.WHXB20111008

(9) Xiao, L. F.; Li, J. P.; Li, Q.; Zhang, L. Z. J. Solid State Electr. 2010, $14,931$.

(10) Zhao, Q.; Xie, Y.; Dong, T.; Zhang, Z. J. Phys. Chem. C 2007, 111 (31), 11598. doi: 10.1021/jp072858h

(11) Liu, J.; Zhang, L.; Shi, S.; Chen, S.; Zhou, N.; Zhang, Z.; Cheng, Z.; Zhu, X. Langmuir 2010, 26 (18), 14806. doi: 10.1021/la102994g

(12) Kobayashi, Y.; Salgueirino-Maceira, V.; Liz-Marzan, L. M. Chem. Mater. 2001, 13 (5), 1630. doi: 10.1021/cm001240g

(13) Liu, R.; Yang, S.; Wang, F.; Lu, X.; Yang, Z.; Ding, B. ACS Appl. Mater. Interfaces 2012, 4, 1537. doi: 10.1021/am201756m

(14) Tierno, P.; Goedel, W. A. J. Phys. Chem. B 2006, 110 (7), 3043. doi: $10.1021 / \mathrm{jp} 054213 \mathrm{~s}$
(15) Chen, M.; Wu, L.; Zhou, S.; You, B. Adv. Mater. 2006, 18 (6), 801.

(16) Jang, I.; Sung, J.; Choi, H.; Chin, I. Synth. Met. 2005, 152 (1-3), 9. doi: 10.1016/j.synthmet.2005.07.087

(17) Li, M.; Lu, Q.; Nuli, Y.; Qian, X. Electrochem. Solid-State Lett. 2007, 10 (8), K33.

(18) Holland, B. T.; Blanford, C. F.; Do, T.; Stein, A. Chem. Mater 1999, 11 (3), 795. doi: 10.1021/cm980666g

(19) (a) Yang, Z.; Niu, Z.; Lu, Y.; Hu, Z.; Han, C. C. Angew. Chem. Int. Edit. 2003, 42, 1943. doi: 10.1002/anie. 200250443

(b) Yang, Z.; Niu, Z.; Lu, Y.; Hu, Z.; Han, C. C. Angew. Chem. 2003, 115 (17), 1987.

(20) Cai, J. Y.; Lü, S.; Sun, W. D. J. Northeast Norm. Univ., Nat. Sci. $E d$. 2009, 41 (2), 135. [蔡建岩, 吕 州, 孙闻东. 东北师大学 报: 自然科学版, 2009, 41 (2), 135.]

(21) (a) Yoon, K.; Yang, Y.; Lu, P.; Wan, D. H.; Peng, H. C.; Masias, K. S.; Fanson, P. T.; Campbell, C. T.; Xia, Y. N. Angew. Chem. 2012, 124 (38), 9681. doi: 10.1002/ange.v124.38 (b) Yoon, K.; Yang, Y.; Lu, P.; Wan, D. H.; Peng, H. C.; Masias, K. S.; Fanson, P. T.; Campbell, C. T.; Xia, Y. N. Angew. Chem. Int. Edit. 2012, 51, 9543.

(22) Huang, Y.; Li, M. Materials Review 2006, 20 (10), 143. [黄 怡, 李 梅. 材料导报, 2006, $20(10), 143$.]

(23) Li, G.; Xi, S. P.; Liu, Z. X.; Huang, Y. E. Spectroscopy and Spectral Analysis 1999, 19 (3), 289. [李 谷, 席世平, 刘振 兴, 黄月娥. 光谱学与光谱分析, 1999, 19 (3), 289.]

(24) Shi, L.; Lin, H. Langmuir 2010, 26 (24), 18718. doi: 10.1021/ la103769d

(25) Zhao, H. Y.; Zhao, Z. Z.; Zhao, Y. F.; Liu, Q. J. Chin. J. Catal. 2010, 31 (1), 44. [ 赵鹤云, 赵忠泽, 赵义芬, 柳清菊. 催化学 报, 2010, $31(1), 44$.] 